\title{
Le développement de la télémédecine en France. Une territorialisation de la politique publique?
}

\section{Maxime Thorigny}

\section{OpenEdition}

Journals

Édition électronique

URL : http://journals.openedition.org/rfst/609

DOI : $10.4000 /$ rfst.609

ISSN : 2492-3672

\section{Éditeur}

Espaces et SOciétés (UMR 6590)

\section{Référence électronique}

Maxime Thorigny, «Le développement de la télémédecine en France. Une territorialisation de la politique publique? », Revue francophone sur la santé et les territoires [En ligne], Ateliers et séminaires, mis en ligne le 25 novembre 2016, consulté le 06 avril 2021. URL : http://journals.openedition.org/rfst/ 609 ; DOI : https://doi.org/10.4000/rfst.609

Ce document a été généré automatiquement le 6 avril 2021.

\section{(i) (2)}

La Revue francophone sur la santé et les territoires est mise à disposition selon les termes de la Licence Creative Commons Attribution - Pas d'Utilisation Commerciale - Partage dans les Mêmes Conditions 4.0 International. 


\title{
Le développement de la télémédecine en France. Une territorialisation de la politique publique?
}

\author{
Maxime Thorigny
}

1 Le développement de la télémédecine en France est consacré par la loi Hôpital, Patient, Santé Territoires (HPST) de 2009, son décret d'application de 2010 définit 5 types d'actes, permettant de réaliser des actes médicaux au moyen « d'un dispositif utilisant les technologies de l'information et de la communication ». La principale innovation réside donc dans un changement organisationnel de la pratique médicale. Les objectifs affichés par les différents ministres de la santé sont clairs: dans un premier temps réduire les inégalités d'accès aux soins par une meilleure coordination à distance des professionnels, dans un second temps maitriser les dépenses de santé en réduisant le transport de patients, en prodiguant le bon soin au bon moment.

De manière concomitante, la planification du secteur de la santé est attribuée aux Agences Régionales de Santé, structures qui sont créées elles aussi par la loi HPST. Elles sont chargées d'établir la politique régionale en matière de santé en formalisant un Programme Régional de Santé qui définit les objectifs à poursuivre, sur la base d'un constat partagé. Différents territoires d'action sont envisagés, leur taille et leur mode de construction sont laissés libres pour chaque ARS. Le Programme Régional de Santé se décline en "schémas» et "programmes» dont le Programme Régional de Télémédecine (PRT), qui définit la politique à tenir en matière de télémédecine. Ce PRT est construit au niveau régional, sur la base d'un guide édité par la Direction Générale de l'Offre de Soins (DGOS) qui fixe la méthodologie et les priorités nationales.

La mise en œuvre des projets va se heurter à plusieurs écueils. Pour le secteur libéral, le manque de clarté juridique sur les outils et les responsabilités des acteurs et le caractère non pérenne des financements sont un frein majeur. Concernant les établissements publics comme privés, la problématique n'est pas seulement financière 
mais se pose aussi en terme de conduite de changement d'un point de vue organisationnel. Enfin, les ARS ont fait des choix techniques différents, en matière de "plateformes de télémédecine » qui sont le support des échanges entre praticiens. Il n'y a pas de logique frontalière dans le cadre de projets extrarégionaux.

4 Afin d'établir une évaluation chiffrée sur l'efficience dans le secteur ambulatoire et avant une généralisation, le Ministère de la santé a retenu 9 régions en 2014 comme territoires expérimentateurs: Alsace, Basse-Normandie, Bourgogne, Centre - Val de Loire, Haute-Normandie, Languedoc-Roussillon, Martinique, Pays de la Loire et Picardie.

5 Cette communication vise donc à analyser la manière dont le territoire est pris en compte et quelles tensions existent entre la politique de déploiement de la télémédecine et la manière dont les acteurs de terrain s'en saisissent.

Dans un premier temps cette communication revient sur l'histoire de la notion de territoire dans le secteur de la santé et la définition du territoire d'action pertinent pour l'Etat (Coldefy et Lucas-Gabrielli (2010 et 2012), Vigneron (2009) et Rican (2004) et Vaillant (2009)). La taille, ainsi que la définition du territoire sont très variables et dépendent de la configuration territoriale elle-même (surface, densité, réseau de communication, besoin et offre de soins). La loi HPST donne le pouvoir aux ARS de définir les territoires avec les outils qu'elles souhaitent, il apparait donc une diversité très importante dans les approches aussi bien en terme de conception que de caractéristiques (rapport de 1 à 14 en terme de population, rapport de 1 à 440 en terme de surface). D'un autre coté l'analyse du contenu des PRT en utilisant le guide de la Direction Générale de l'offre de Soins du Ministère de la santé comme grille de lecture révèle que toutes les priorités et les recommandations n'ont pas été respectées et que l'on trouve des initiatives régionales. Nous nous interrogeons donc sur la prise en compte des territoires et la manière dont ils sont appréhendés dans la politique de télémédecine des ARS? Il semble que le territoire soit considéré comme un réseau entre praticiens de premier et second recours de manière formelle, car c'est sur cette dimension que se développe les projets de télémédecine et non en matière d'adéquation entre une offre et un besoin de soins spatialement localisé.

7 Dans un second temps nous nous attachons à étudier les mécanismes de l'expérimentation article 36, par la lecture du New Public Management (Pyun (2013) et Boyer (1997)) et le caractère performatif du discours de la tutelle (Jany-Catrice 2012), en imposant un cadre réglementaire très strict avec une contractualisation croisée entre l'ARS, la Caisse Primaire d'Assurance Maladie et les professionnels de santé. De la même manière, le tarif des expérimentations est jugé insuffisant par les professionnels ainsi que le périmètre (plaies chroniques et complexes). Le ministère n'a pas laissé de marge de manœuvre aux ARS quant à l'adaptabilité de l'expérimentation aux besoins des territoires ou aux structures existantes à l'exception du choix des prestataires des solutions des "plateformes de télémédecine ». A ce titre, nous montrons qu'il n'y a pas de logique frontalière claire, car les choix techniques ont été faits par région sur le fondement de marché publics. Plusieurs industriels se partagent le marché au niveau national, il n'existe pas encore de norme opposable concernant les formats d'échanges de données ce qui posera dans le futur proche des problèmes d'interopérabilité des systèmes dans une perspective de déploiement national (figure 1). Nous arrivons ici à une conclusion paradoxale, la télémédecine a pour vocation à s'affranchir des contraintes de localisation des professionnels de santé, mais sa politique de 
déploiement amène à renforcer les frontières régionales en rendant difficile voire impossible la coopération interrégionale. A ce jour, cette expérimentation a donné lieu à moins de 100 actes facturés depuis 2014. Le ministère de la santé, conscient des problèmes rencontrés, propose donc des modifications majeures de l'expérimentation : élargissement à l'ensemble des Affections Longues Durées, nouveaux tarifs plus attractifs, évaluations réalisées par la Haute Autorité de Santé à partir des bases de données de l'assurance maladie et suppression de la contractualisation qui pose la question de la place des ARS dans le suivi de ces expérimentations.

Enfin, nous montrons quels sont les déploiements en cours des projets de télémédecine et comment ils répondent à des besoins en matière de santé des territoires, où la coproduction entre les différents acteurs, établissements, professionnels et tutelle, fonctionne autour d'un cadre institutionnel stable. Nous prendrons deux exemples nationaux, le déploiement du téléAVC et de la téléradiologie.

Le téléAVC consiste à la consultation à distance d'un chirurgien neurologue préalable à la thrombolyse médicamenteuse ${ }^{1}$, l'enjeu est de proposer un diagnostic dans les $4 \mathrm{~h}$ après la survenue des symptômes. Des Unités Neuro-Vasculaires sont dédiées à ces urgences particulières, cependant leur répartition territoriale est inégale et l'accessibilité est très variable. Le but est qu'un centre d'urgence traditionnel accessible plus rapidement, puisse bénéficier de l'expertise d'une UNV sans déplacer le patient. Plusieurs types d'organisations définies par les professionnels eux-mêmes à deux niveaux, au niveau de chaque équipe, centre requérant et requis, mais aussi entre établissements. 5 types d'organisations ont été synthétisés par l'Agence Nationale d'Appui à la Performance des établissements sanitaires et sociaux (ANAP) permettent d'adapter le fonctionnement interne des établissements et les relations interétablissement (ANAP 2014).

De la même façon le déploiement de la téléradiologie s'est fait dans une logique de coopération entre plusieurs sites et plusieurs professions, en effet, il n'y a pas besoin de professionnels médicaux dans le centre requérant, un manipulateur d'électroradiologie est suffisant. Le droit reconnait cette pratique par la division en forfaits des actes techniques et intellectuels qui a été construite à l'aide des sociétés savantes et des représentants syndicaux.

11 Dès lors, nous mettrons en évidence que la coordination (Batifoulier 1992, Béjan 1999) entre les acteurs passe par la définition d'une convention partagée dans le sens normatif de la théorie des conventions. Celle-ci ne peut être qu'une co-construction territorialement cohérente et ne peut donc pas être définie ex-ante par le référentiel politique.

12 Le développement de la télémédecine en France passe par deux vecteurs: l'expérimentation nationale dite «article 36 » qui porte sur 9 anciennes régions et la politique régionale déployée par les ARS, sur la base de recommandations du Ministère de la santé. Le déploiement sur le terrain est très variable d'une région à l'autre car les objectifs des ARS sont différents. De même la définition qu'elles font du territoire ne repose pas sur les mêmes normes, ce qui aboutit à des espaces de projets de tailles variables.

13 Nous montrons qu'il y a un problème de définition de la politique publique en santé : d'un côté la loi HPST consacre les ARS et la région comme territoire de définition de la politique de santé, de l'autre les objectifs sont cadrés par le Ministère de la santé, sans 
tenir compte des spécificités des territoires. A ce titre, la télémédecine est un bon exemple de conflit de représentations et d'objectifs entre l'échelon national et régional.

L'expérimentation nationale se révèle être pour le moment un échec par l'imposition de contrats et de modes d'organisation, ne laissant pas la possibilité aux acteurs de s'organiser entre eux à leur convenance, en respectant les organisations déjà mises en place. En ce sens l'imposition de contrats nationaux ne permet pas la co-construction et la définition d'une convention partagée entre acteurs. Alors qu'il existe des exemples d'utilisation de la télémédecine, notamment concernant le traitement de l'AVC en urgence ou plus généralement la téléradiologie, qui s'adaptent aux pratiques des professionnels de santé en respectant les réseaux de recours professionnels déjà établis.

On peut se demander si la tutelle n'est pas en train de changer sa manière de déployer la politique de télémédecine. En effet, la réforme des Groupements Hospitaliers de Territoire (GHT), qui organise les établissements en réseaux de recours afin d'organiser la gradation des soins, institutionnalise la télémédecine comme une nouvelle norme de coopération entre professionnels d'établissements d'un même GHT avec pour objectifs de réduire les coûts de transports et de personnels. On s'oriente donc vers une coconstruction aussi bien en interne qu'en externe des établissements.

\section{BIBLIOGRAPHIE}

ANAP. (2014), « La télémédecine en action : prise en charge de l'AVC -Support d'aide au déploiement régional ».

BATIFOULIER P. (1992), « Le rôle des conventions dans le système de santé », Sciences Sociales et Santé

BEJAN S. (1999), «De nouvelles théories en économie de la santé : fondements, oppositions et complémentarités, Politiques et management public

BOYER R, (1997), « Evolution des modèles productifs », Paris, CEPREMAP

COLDEFY M. et LUCAS-GABRIELLI V. (2010), « Les territoires de santé : des approches régionales variées de ce nouvel espace de planification », Pratiques et Organisation des Soins, CNAMTS

COLDEFY M. et LUCAS-GABRIELLI V. (2012), « Le territoire, un outil d'organisation des soins et des politiques de santé ?", Questions d'économie de la Santé, IRDES

JANY-CATRICE F. (2010), « La performance totale : nouvel esprit du capitalisme? », Villeneuve d'Ascq, Presses universitaires du Septentrion

PYUN H. (2013), « Hybridation du New public Management : cas de gestion des administrateurs territoriaux en France, Management \& Avenir

RICAN S. et VAILLANT Z. (2009), « Territoires et santé : enjeux sanitaires de la territorialisation et enjeux territoriaux des politiques de santé », Sciences sociales et Santé.

VIGNERON E. (2004), « Des territoires de santé pour quoi faire? » Soins Cadres 


\section{NOTES}

1. Il existe deux types d'Accident Vasculaire Cérébral. Les AVC ischémiques qui se présentent sous la forme de l'obstruction d'un vaisseau sanguin et qu'il faut thrombolyser afin de permettre une circulation sanguine correcte, ce procédé consiste en l'injection d'un médicament fluidifiant le sang. Le deuxième type d'AVC est l'AVC hémorragique qui nécessite une intervention chirurgicale. Si la décision est prise de thrombolyser un AVC hémorragique le traitement aggravera significativement l'hémorragie cérébrale, c'est pourquoi le protocole impose l'avis d'un neurologue avant toute opération.

INDEX

Index géographique : France

Mots-clés : télémédecine, territorialisation, politiques publiques

\section{AUTEUR}

MAXIME THORIGNY

REGARS - EA 6292, Université de Reims Champagne-Ardenne 